\title{
Depletion of Janus kinase-2 promotes neuronal differentiation of mouse embryonic stem cells
}

\author{
Mihee Oh ${ }^{1}$, Sun Young Kim ${ }^{1}$, Jeong-Su Byun ${ }^{1}$, Seonha Lee ${ }^{1,3}$, Won-Kon Kim ${ }^{2,3}$, Kyoung-Jin Oh ${ }^{2,3}$, Eun-Woo Lee ${ }^{2,3}$, \\ Kwang-Hee Bae ${ }^{2,3}$, Sang Chul Lee $e^{2,3}$ E Baek-Soo Han ${ }^{1,2,3, *}$ \\ ${ }^{1}$ Biodefense Research Center, ${ }^{2}$ Metabolic Regulation Research Center, Korea Research Institute of Bioscience and Biotechnology, Daejeon \\ 34141, ${ }^{3}$ Department of Functional Genomics, University of Science and Technology (UST) of Korea, Daejeon 34113, Korea
}

\begin{abstract}
Janus kinase 2 (JAK2), a non-receptor tyrosine kinase, is a critical component of cytokine and growth factor signaling pathways regulating hematopoietic cell proliferation. JAK2 mutations are associated with multiple myeloproliferative neoplasms. Although physiological and pathological functions of JAK2 in hematopoietic tissues are well-known, such functions of JAK2 in the nervous system are not well studied yet. The present study demonstrated that JAK2 could negatively regulate neuronal differentiation of mouse embryonic stem cells (ESCs). Depletion of JAK2 stimulated neuronal differentiation of mouse ESCs and activated glycogen synthase kinase $3 \beta$, Fyn, and cyclin-dependent kinase 5. Knockdown of JAK2 resulted in accumulation of GTPbound Rac1, a Rho GTPase implicated in the regulation of cytoskeletal dynamics. These findings suggest that JAK2 might negatively regulate neuronal differentiation by suppressing the GSK-3 $\beta$ / Fyn/CDK5 signaling pathway responsible for morphological maturation. [BMB Reports 2021; 54(12): 626-631]
\end{abstract}

\section{INTRODUCTION}

Janus kinase 2 (JAK2) is a non-receptor somatic tyrosine kinase belonging to the Janus family of kinases frequently involved in cytokine and growth factor signaling pathways (1). Binding of JAK2 to the associated receptor can lead to activation through autologous and trans phosphorylation at tyrosine 1007/1008 (1). In turn, activation of JAK2 triggers a variety of downstream signaling cascades by phosphorylating specific tyrosine residues on target proteins.

Expression of JAK2 has been found in numerous tissues (2).

*Corresponding author. Tel: +82-42-879-8438; Fax: +82-42-879-8596; E-mail: bshan@kribb.re.kr

https://doi.org/10.5483/BMBRep.2021.54.12.154

Received 30 October 2021, Revised 15 November 2021, Accepted 15 November 2021

Keywords: Cyclin-dependent kinase 5 (CDK5), Embryonic stem cell, Glycogen synthase kinase $3 \beta$ (GSK3 $\beta$ ), Janus kinase-2 (JAK2), Neurogenesis, Neuronal differentiation
In addition, many studies have shown that JAK2 is localized in the cytoplasm, endoplasmic reticulum, and nucleus (3), suggesting its additional physiological and pathophysiological signaling functions $(4,5)$. Despite critical functions of JAK2 in various organs and tissues are well-known, little is known about JAK2 functions in nervous system.

Recent studies have implicated the JAK/STAT pathway in leptin-mediated neuroprotection and in central control of food intake (6). Others have reported that JAKISTAT pathway activity can drive astrocyte reactivity and memory deficits in Alzheimer's disease models (7). However, specific cellular and molecular mechanisms regulated by JAK/STAT signaling in neurons are not well studied yet.

The present study showed that depletion of JAK2 enhanced neuronal differentiation of $\mathrm{J} 1$ mouse embryonic stem cells (ESCs) as evidenced by upregulated neuronal marker expression and neurite outgrowth. Results of this study suggest that JAK2 normally suppresses neuronal differentiation from ESCs by negatively regulating the GSK-3 $\beta$ beta/Fyn/CDK5 signaling pathway.

\section{RESULTS}

Depletion of JAK2 enhances neurite outgrowth

To analyze expression levels of JAK2 during neural differentiation, both JAK2 and phosphorylated (p)-JAK2 expression levels were examined by Western blotting. Both proteins were downregulated progressively during culture under neuronal differentiation conditions (Fig. 1A), with p-JAK2 expression being significantly lower in differentiated ESCs than in undifferentiated ESCs (Fig. 1B). These findings suggest that JAK2 activation normally serves to limit the progression of neuronal differentiation and that its downregulation is an important event in the progression of differentiation.

To confirm this effect of JAK2 downregulation on neuronal differentiation, we examined phenotypic changes following shRNA-mediated JAK2 knockdown. Expression levels of JAK2 mRNA and protein were dramatically decreased in cells transfected with shJAK2 compared to those in cells transfected with scrambled shRNA (Fig. 2A-C). Furthermore, JAK2 depletion markedly increased the neuronal differentiation efficiency of J1 ESCs by day 3 of induction as evidenced by enhanced outgrowth of 
neurites and immunostaining for a neuronal differentiation marker TUJ1 (Fig. 2D). Expression levels of neuron-specific marker proteins MAP2, synapsin1, and synaptophysin as well as TUJ1 were also increased in JAK2 knockdown cells during differentiation culture (Fig. 2E, F). However, JAK2 knockdown had no effect on cell survival on day 3 as evidenced by undetectable expression of apoptosis effector cleaved caspase-3 (Fig. 2G).

We also examined effects of the cell permeable JAK2 inhibitor SD-1029 (8). Consistent with gene knockdown results, expression levels of neuronal markers were upregulated compared to those in vehicle-treated cells under SD-1029 treatment condition (Fig. 2H). Collectively, these results demonstrate that inhibiting JAK2 activity or expression can promote neuronal differentiation.

Next, we investigated whether the enhancement of differentiation by JAK2 knockdown could be reversed by reintroduction of JAK2 using an overexpression vector. As shown in Fig. 2l, effects of JAK2 knockdown, including neurite outgrowth and altered expression of neuron-specific marker proteins, were reversed to levels in normally differentiated neurons by JAK2 transfection (Fig. 2I).

\section{Depletion of JAK2 positively regulates the activity of GSK-3 $\beta$} and Fyn kinase

To identify signaling pathways mediating enhanced neuronal differentiation following JAK2 knockdown, we first screened for changes in expression levels of signaling molecules known to participate in JAK2 signaling cascades. Previous studies have demonstrated that GSK-3 $\beta$ is a common signaling molecule in JAK2 cascades either as a downstream target, upstream effector, or by acting cooperatively $(9,10)$. To address the association between GSK-3 $\beta$ and JAK2 during neuronal differentiation, we analyzed the activity of GSK-3 $\beta$ in JAK2-deficient cells on differentiation day 3 . We found that GSK3 $\beta$ phosphorylation at Ser9
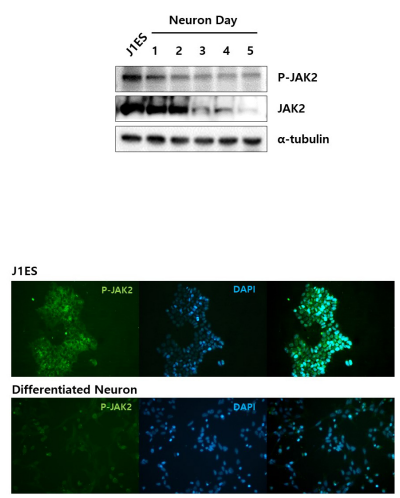

Fig. 1. JAK2 is downregulated during neuronal differentiation. (A) Expression levels of JAK2 and phosphorylated (p)-JAK2 in J1 embryonic stem cells (ESCs) at various time points during differentiation culture as analyzed by immunoblotting. (B) Immunofluorescence analysis of $\mathrm{J} 1$ ESCs and differentiated neurons. Phosphorylated JAK2 was stained green. was decreased while phosphorylation at Tyr216 was increased in JAK2 knockdown cells (Fig. 3A). Phosphorylation at Y216 is known to activate GSK-3 $\beta$, suggesting that GSK-3 $\beta$ activation is required for the differentiation-promoting effect of JAK2 depletion $(9,10)$. Also, consistent with a contribution of JAK2-GSK3 $\beta$ signaling to neuronal differentiation, treatment with a GSK inhibitor $\mathrm{LiCl}$ for $12 \mathrm{~h}$ decreased expression levels of neuronal differentiation markers in JAK2-knockdown ESC-derived neurons to levels measured in untreated JAK2-knockdown neurons (Fig.
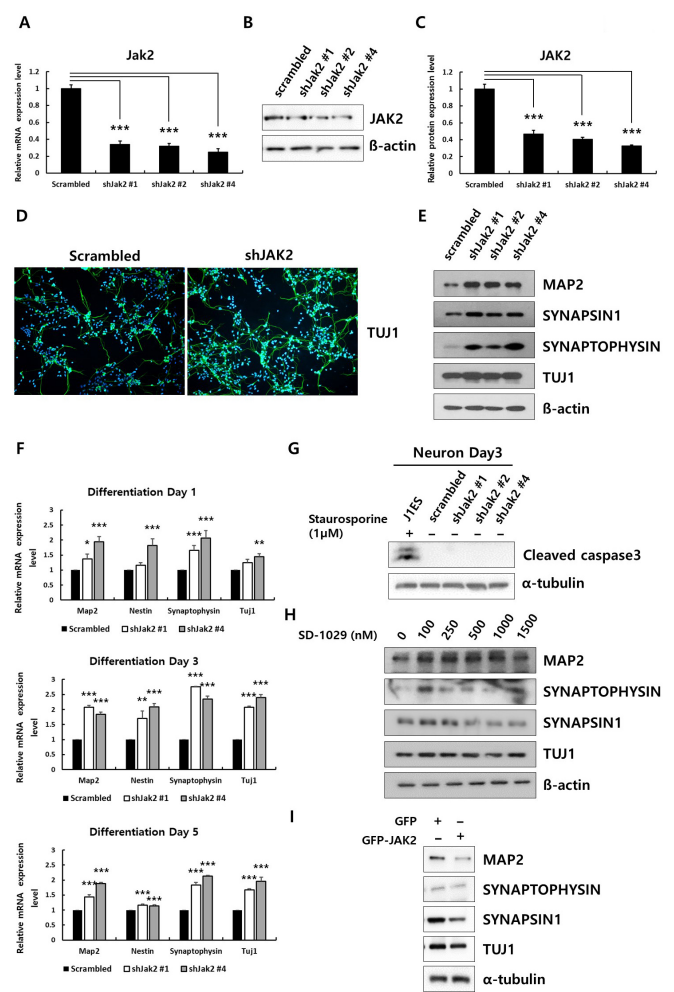

Fig. 2. Depletion of JAK2 enhances neurite outgrowth. (A-C) J1 ESCS were infected with lentivirus encoding scrambled or JAK2-targeted small hairpin RNA (shRNA) and cultured under differentiation conditions. Knockdown efficiency was confirmed by semi-quantitative polymerase chain reaction ( $\mathrm{PPCR}$ ) (A) and immunoblotting (B) after three days of differentiation culture. (C) Expression levels of JAK2 as determined by densitometry. (D) Immunofluorescent images of ESCs infected with either a scrambled or JAK2-targeted shRNA and cultured under neuronal differentiation conditions. Representative images were acquired on day 3. The neuronal marker TUJ1 was stained green. $(E, F)$ Expression levels of neuron-specific marker proteins analyzed by immunoblotting $(\mathrm{E})$ and $\mathrm{qPCR}(\mathrm{F})$ in control and JAK2-knockdown J1 ESCs after 3 days of neuronal differentiation culture. (G) Cleaved caspase 3 expression in differentiated J1 ESC-derived neurons as assessed by immunoblotting. $(\mathrm{H})$ Expression levels of neuronal markers in J1 ESCs treated for 24 with the JAK2 inhibitor SD-1029 or vehicle as estimated by western blotting. (I) ESCs infected with GFP or GFP-JAK2 WT vector and grown in differentiation culture. Whole-cell lysates were prepared on day 3 for western blot analysis of neuron-specific markers. All results are expressed as mean \pm SD. Treatment group means were compared by Student's t-test $(\mathrm{n}=3 ; * \mathrm{P}<0.05 ; * * \mathrm{P}<0.01 ; * * * \mathrm{P}<0.005)$. 

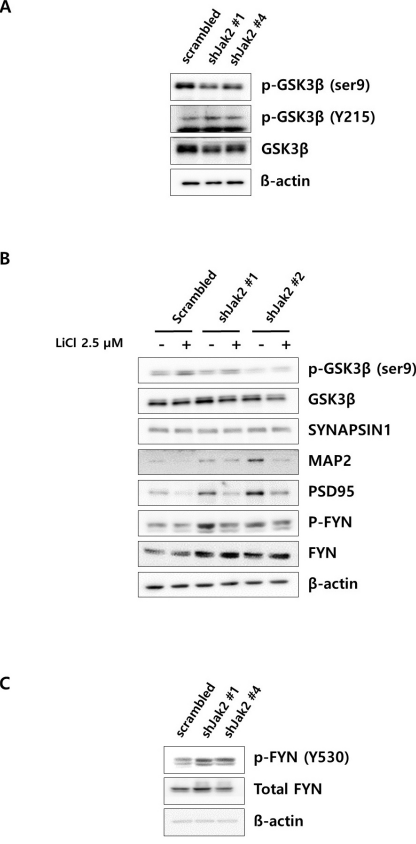

Fig. 3. Depletion of JAK2 upregulates activities of GSK3beta and Fyn. (A) JAK2 knockdown cells were induced to differentiate into neuronal cells for three days and subjected to immunoblotting for GSK3beta and Fyn. (B) JAK2 knockdown cells were induced to differentiate into neuronal cells for 2 days and then treated with $2.5 \mu \mathrm{M}$ of the GSK3beta inhibitor $\mathrm{LiCl}$ or saline vehicle for $24 \mathrm{~h}$. Whole-cell lysates were prepared on day 3 for Western blot analysis. (C) JAK2 knockdown cells were induced to differentiate into neuronal cells for three days and then examined for GSK3beta and Fyn expression levels by immunoblotting.

3B). These findings suggest that JAK2 can promote differentiation in part by negatively regulating GSK-3 $\beta$ activity.

It is likely that GSK-3 $\beta$ functions upstream of Fyn because nuclear accumulation of Fyn is dependent on GSK-3 $\beta$ activity (11). Fyn is a membrane-associated tyrosine kinase expressed in the CNS. It is a well-known regulator of T cell receptor signaling, cell division, and cell adhesion (12). To examine potential involvement of Fyn in JAK2-GSK-3 $\beta$ signaling during neuronal differentiation, Fyn expression levels were compared between JAK2-deficient ESCs and control ESCs on day 3 in differentiation culture. Like GSK-3 $\beta$, phosphorylation of Fyn was greater in JAK2 knockdown cells when compared to control cells (Fig. 3C). Furthermore, treatment with $\mathrm{LiCl}$ reduced Fyn phosphorylation in JAK2 knockdown cells under differentiation culture conditions (Fig. 3B), suggesting that GSK-3 $\beta$ could act as an intermediate signal for Fyn activation following JAK2 knockdown.

\section{JAK2 knockdown positively regulates CDK5}

To identify additional downstream signaling mechanisms involved in the promotion of neuronal differentiation by JAK2 depletion, we examined expression levels and activities of known downstream targets of GSK-3 $\beta$-Fyn. Previous studies have demonstrated

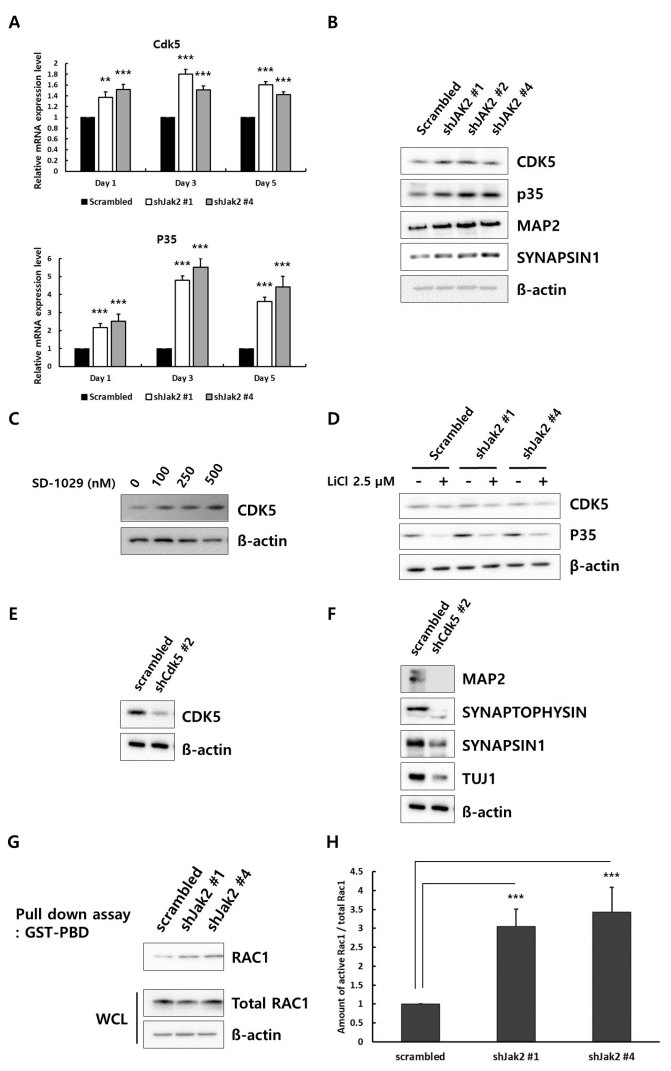

Fig. 4. JAK2 knockdown upregulates CDK5. (A, B) JAK2 knockdown cells were induced to differentiate into neuronal cells for three days and expression levels of CDK5 and P35 were analyzed by semi-quantitative polymerase chain reaction (qPCR) (A) and immunoblotting (B). (C) J1ES cells were induced to differentiate into neuronal cells for 2 days and then treated with SD-1029 (indicated concentration) or DMSO for $24 \mathrm{~h}$. Expression levels of CDK5 and actin were analyzed by Western blot. (D) JAK2 knockdown cells were induced to differentiate into neuronal cells for 2 days and then incubated with $2.5 \mu \mathrm{M} \mathrm{LiCl}$ or saline for $24 \mathrm{~h}$. Whole-cell lysates were prepared on day 3 for western blot analysis of CDK5, P35, and actin expression. (E) J1 ESCs were infected with lentivirus vector encoding scrambled shRNA or shCDK5 and differentiated into neurons. Knockdown efficiency was analyzed by immunoblotting after three days of differentiation culture. (F) CDK5 knockdown cells were induced to differentiate into neuronal cells for three days and then subjected to Western blot analysis of neuron specific markers. (G) JAK2 knockdown cells were induced to differentiate into neuronal cells for three days and then GST-fused Pak binding domain was used for pull-down assay to determine GTP-bound active Rac1 protein levels, while a parallel western blot analysis of total cell lysate was performed to determine total Rac1 expression level. $(H)$ Quantitation by densitometry. Data are expressed as mean \pm SD. Treatment group means were compared by Student's t-test $\left({ }^{*} \mathrm{P}<0.05\right.$; $* * P<0.01 ; * * * P<0.005)$.

that activated Fyn is associated with CDK5 and that Fyn can promote CDK5 activation via Tyr15 phosphorylation $(13,14)$. Consistent with these findings, expression levels of CDK5 were elevated in JAK2 knockdown cells as well as expression levels of the brain-specific CDK5-related protein and activator P35 
(Fig. 4A, B) $(15,16)$. The expression level of CDK5 was also increased after cells were exposed to a JAK2 inhibitor SD-1029 (Fig. 4C). Furthermore, expression levels of both proteins were reduced by GSK-3 $\beta$ inhibitor $\mathrm{LiCl}$ (Fig. 4D). Therefore, CDK5 appears to be a direct downstream target of the JAK2-GSK-3 $\beta$ Fyn cascade.

It has been shown that the CDK5/p35 complex can regulate neurite outgrowth in cultured rat hippocampal neurons (16), suggesting that the observed CDK5 upregulation under JAK2 knockdown is also required for the pro-differentiation response. To test this notion, we generated J1 ESCs stably expressing shCDK5 and confirmed that CDK5 expression was efficiently suppressed (Fig. 4E). As predicted, CDK5 knockdown completely inhibited neurite outgrowth in JAK2 knockdown ESCs during differentiation culture (Fig. 4F).

\section{JAK2 knockdown enhances Rac1 activation}

We then examined potential Fyn-CDK5-dependent mechanisms for neurite outgrowth in JAK2-depleted neurons. The Rho family of small GTPases (Rac1 and CDC42) is involved in cytoskeletal rearrangement $(17,18)$. Moreover, both Rac1 and CDC42 have been implicated in neurite outgrowth from neuronal cells (19). A previous study has also reported that CDK5/P35 can phosphorylate serine/threonine kinase Pak1 in a RacGTP-dependent manner, which in turn can regulate neural morphology via phosphorylation of various cytoskeletal proteins (20). Thus, we investigated whether Rac1 was involved in neurite outgrowth caused by JAK2 knockdown. Results showed that lysates from JAK2 knockdown ESCs contained greater amounts of endogenous GTP-bound Rac1 compared to those from control ESCs under the same culture conditions as revealed by GST-PAK pull-down assays (Fig. 4G, H).

\section{DISCUSSION}

The JAK2 tyrosine kinase is implicated in numerous developmental, physiological, and pathological processes (21) through its associations with cytokine and growth factor receptors (2). However, many fundamental questions regarding JAK2 remain unclear, including its role in nervous system development, adult neurogenesis, appetite control, and neuro-inflammation. In this study, we found that JAK2 knockdown markedly enhanced the neuronal differentiation capacity of ESCs. This suggests that JAK2 normally suppresses neuronal differentiation and maturation.

There is accumulating evidence that JAK2 is a multifunctional signaling factor in the central nervous system. JAK2 is highly expressed in the developing brain, particularly in the early embryonic stages. It then gradually decreases with age (22). Our results were consistent with these developmental studies as expression levels of JAK2 and phosphorylated JAK2 in J1 ESCs were gradually decreased during differentiation culture and inversely related to neuronal maturation.

The strong association of JAK2 with cytokine receptors suggests potential functions of JAK2 in neurodegenerative/neuroinflammatory disorders. The JAK/STAT3 pathway is activated in reactive astrocytes in several mouse and nonhuman primate models of Alzheimer's disease (AD) and Huntington's disease (HD) (23). Moreover, an APP/PS1 mouse model of AD shows reduced neuronal differentiation and markedly increased astrocytic differentiation compared to WT controls (24). Intriguingly, treatment with JAK2 inhibition enhanced neuronal differentiation capacity but inhibited the production of astrocytes, further supporting our findings that JAK2 could negatively regulate neuronal differentiation (24). The same group has also found that the JAK2/STAT3 pathway is activated in the hippocampus and that JAK2/STAT3 inhibition can rescue cognitive deficits and adult hippocampal neurogenesis in APP/PS1 mice (24). Elucidation of the signaling pathways linking JAK2 to neurogenesis may help the development of treatments for neurodegenerative and neuropsychiatric disorders.

We also demonstrated that JAK2 deficiency and neuronal differentiation were associated with increased activity of GSK-3 $\beta$, a kinase known to promote neuronal survival $(25,26)$. Overexpression of constitutively active GSK-3 $\beta$ in neurons can trigger differentiation $(25,26)$. Alternatively, other reports have found that GSK-3 $\beta$ activity can promote neurite growth (27). Similarly, GSK-3 $\alpha / \beta$ double knockout ESCs exhibit severely impaired differentiation capacity primarily due to hyper-activation of the Wnt signaling pathway (28). Additionally, expression of mutant GSK-3 $\beta$ and RNAi-mediated GSK-3 knockdown can disrupt neuronal polarity in primary culture (29). Knock-in mice expressing GSK-3 $\alpha / \beta$ (Ser21/9Ala) show decreased neurogenesis and induced abnormal behavior (30). This suggests that low GSK-3 $\beta$ activity can promote proliferation and prevent differentiation. However, there are conflicting data regarding neuronal differentiation. It has been reported that GSK-3 $\beta$ can promote neural differentiation of PC12 cells (31). The most parsimonious conclusion is that the influence of GSK-3 3 on neuronal differentiation is highly dependent on substrate expression. Substrates negatively regulated by GSK-3 $\beta$ are generally involved in enhancing proliferation and/or survival, whereas substrates positively regulated by GSK-3 3 are required for mature neuronal function. Thus, they are primarily expressed in differentiated neurons (32). Substrates negatively regulated by GSK-3 $\beta$ include proliferation-related transcription factors, while substrates positively regulated by GSK-3 $\beta$ include cytoskeletonrelated factors, which could in turn mediate morphological maturation.

Expression levels of CDK5/p35 and p-CDK5 were also increased in JAK2-knockdown cells differentiated into neurons. Furthermore, CDK5 knockdown abrogated neurite outgrowth under JAK2 deficiency. While CDK5 phosphorylation by Fyn kinase and its subsequent activity are expected, upstream pathways increasing CDK5 expression following JAK2 knockdown are unclear. Expression of CDK5 is upregulated through the Erk $1 / 2$ pathway and transcription factors Fos and CAMP-responsive element binding protein (CREB) (33). The transcription of $C d k 5$ is also stimulated by the FosB gene product $\delta$ FosB and by a member of the Jun family of transcription factors (34). Future studies are needed to confirm the involvement of these pathways in CDK5 upregulation under JAK2 deficiency and during neuronal differentiation. 
In conclusion, the current results indicated that JAK2 negatively could regulate neuronal differentiation. Depletion of JAK2 stimulated neuronal differentiation capacity through sequential activation of GSK-3 $\beta$, Fyn, and CDK5. Thus, we identified a previously unknown regulatory mechanism for neurogenesis mediated by JAK2 in ESCs. Future work is needed to determine whether the same signaling mechanisms operate in vivo. Studies on how JAK2 and its downstream effectors regulate neurogenesis may help develop novel treatment strategies for neurological diseases such as Alzheimer's disease, Parkinson's disease, and neuropsychiatric conditions such as major depression.

\section{MATERIALS AND METHODS}

\section{Cell culture and neuronal differentiation}

The mESC line, J1 ES [American Type Culture Collection (ATCC), Manassas, VA], derived from strain 129s4/Jae was routinely maintained on gelatin (Millipore, Billerica, MA) coated- dishes in ESC medium with $1 \times 10^{3} \mathrm{U} / \mathrm{ml}$ of leukemia inhibitory factor (LIF) (Millipore) at $37^{\circ} \mathrm{C}$ in a humidified atmosphere with $5 \% \mathrm{CO} 2$.

Cells were differentiated in medium without LIF as described previously (35). All trans-RA (10 $\mu \mathrm{M})$ (Sigma-Aldrich, St. Louis, $\mathrm{MO}$ ) was added every two days from day 4 to 6 . At day 8 , EBs were trypsinized and cells were plated on poly-D lysine/laminin (Sigma-Aldrich)-coated dishes in DMEM medium containing $\mathrm{N} 2$ supplement (Invitrogen) and $20 \mathrm{ng} / \mathrm{ml}$ bFGF (Invitrogen).

\section{Construction of lentiviral vectors and transduction}

For stable expression of GFP-tagged wild type JAK2, ESCs were infected with pLentiX-GFP-c1 vector (Clontech Laboratories, Mountain View, CA) encoding GFP-tagged JAK2. The concentrated virus was used to infect cells in the presence of $8 \mu \mathrm{g} / \mathrm{ml}$ polybrene (Sigma-Aldrich). Infected cells were selected using a FACSAria cell sorter (BD biosciences, San Jose, CA).

For stable JAK2 or CDK5 knockdown, ESCs were transfected with vector encoding a puromycin resistance gene and short hairpin (sh)RNA sequences targeting JAK2 (shJAK2) or CDK5 (shCDK5) (Sigma-Aldrich). shJAK2 or shCDK5 transduced cells were selected by repeated passage for 10 days in medium containing $10 \mu \mathrm{g} / \mathrm{ml}$ puromycin (Invitrogen).

\section{RNA extraction and real-time polymerase chain reaction}

Total RNA was extracted from the cultured cells using the Trizol RNA reagent (Qiagen, Hilden, Germany). RNA extraction carried out following the manufacturer's instructions. First-stranded complementary DNA (cDNA) was synthesized following the manufacturer's instructions.

\section{Western blot analysis}

Cells were washed with ice-cold phosphate buffered saline (PBS), harvested, and lysed using NP40 lysis buffer [ $1 \%$ Nonidet P-40, $150 \mathrm{mM} \mathrm{NaCl}, 10 \%$ glycerol, $2 \mathrm{mM}$ EDTA, and $20 \mathrm{mM}$ Tris- $\mathrm{HCl}(\mathrm{pH} 8)]$ containing protease inhibitors (cOmplate mini EDTA-free; Roche, Penzberg, Germany). Proteins were separated by sodium dodecyl sulfate-polyacrylamide gel electrophoresis (SDS-PAGE) and transferred to polyvinylidene fluoride membranes (Millipore).

The primary antibodies used were anti-MAP2 (Abcam, Cambridge, UK), anti-TUJ1 (Cell Signaling, Danvers, MA, USA), antiSYNAPTOPHYSIN (Cell Signaling), anti-SYNAPSIN1 (Cell Signaling), anti-JAK2 (Abcam), anti-pJAK2 (Abcam), anti-CDK5 (Cell Signaling), anti-FYN (Abcam), anti-pFYN ( Abcam), anti-GSK-3 $\beta$ (Cell Signaling), anti-pGSK-3 $\beta$ (S9) (Cell Signaling), anti-pGSK-3 $\beta$ (Y215) (Abcam), anti-RAC1 (Cell Signaling), anti- $\beta$-actin (Sigma-Aldrich), and anti- $\alpha$-tubulin (Sigma-Aldrich) as the gel loading control.

\section{Small GTPase activity assay}

Cells were harvested with ice-cold lysis buffer (25 mM HEPES [pH 7.5], $150 \mathrm{mM} \mathrm{NaCl}, 1 \%$ Nonidet P-40, $10 \mathrm{mM} \mathrm{MgCl}$, 1 $\mathrm{mM}$ EDTA, $10 \%$ glycerol, $10 \mathrm{mM} \mathrm{NaF}, 1 \mathrm{mM} \mathrm{Na}_{3} \mathrm{VO}_{4}$, and complete protease inhibitor mixture). Total cell lysates were clarified by centrifugation, and incubated overnight at $4^{\circ} \mathrm{C}$ with GST-PBD (p21-binding domain from PAK for Rac1 and Cdc42) immobilized on GST beads. Bound small GTPases were separated by $15 \%$ SDS-PAGE and detected by western blotting with antibodies against Rac1.

\section{Immunofluorescence}

Cells were rinsed with PBS and fixed in $4 \%$ paraformaldehyde (Invitrogen) for $15 \mathrm{~min}$ at room temperature. After washing with PBS, the fixed cells were permeabilized and blocked with $0.1 \%$ Triton ${ }^{\circledR}$ X-100 (Sigma) diluted in $1 \times$ Western Blocking Reagent Solution (Roche) for $1 \mathrm{~h}$ at room temperature. Cells were incubated overnight with primary antibodies at $4^{\circ} \mathrm{C}$ and incubated with Alexa 488- or Alexa 594-conjugated secondary antibodies (Invitrogen) at room temperature for $1 \mathrm{~h}$. Fluorescence images were captured using a Leica microscope (Leica Microsystems, Wetzlar, Germany).

\section{Statistical analysis}

Group means were compared by Student's t-test. A P $<0.05$ (two-tailed) was regarded as statistically significant $(* \mathrm{P}<0.05$, $* * \mathrm{P}<0.01, * * * \mathrm{P}<0.001)$.

\section{ACKNOWLEDGEMENTS}

This work was supported by grants from the Defense Acquisition Program Administration (ADD-911255201).

\section{CONFLICTS OF INTEREST}

The authors have no conflicting interests.

\section{REFERENCES}

1. Leonard WJ and O'Shea JJ (1998) Jaks and STATs: biological implications. Annu Rev Immunol 16, 293-322

2. Behrmann I, Smyczek T, Heinrich PC et al (2004) Janus 
kinase (Jak) subcellular localization revisited: the exclusive membrane localization of endogenous Janus kinase 1 by cytokine receptor interaction uncovers the Jak.receptor complex to be equivalent to a receptor tyrosine kinase. J Biol Chem 279, 35486-35493

3. Ram PA and Waxman DJ (1997) Interaction of growth hormone-activated STATs with SH2-containing phosphotyrosine phosphatase SHP-1 and nuclear JAK2 tyrosine kinase. J Biol Chem 272, 17694-17702

4. Ihle JN and Gilliland DG (2007) Jak2: normal function and role in hematopoietic disorders. Curr Opin Genet Dev $17,8-14$

5. Lee $\mathrm{H}$, Jeong AJ and Ye SK (2019) Highlighted STAT3 as a potential drug target for cancer therapy. BMB Rep 52, 415-423

6. Tups A (2009) Physiological models of leptin resistance. J Neuroendocrinol 21, 961-971

7. Chiba T, Yamada M and Aiso S (2009) Targeting the JAK2/STAT3 axis in Alzheimer's disease. Expert Opin Ther Targets 13, 1155-1167

8. Duan Z, Bradner JE, Greenberg E et al (2006) SD-1029 inhibits signal transducer and activator of transcription 3 nuclear translocation. Clin Cancer Res 12, 6844-6852

9. Nagao T, Kurosu T, Umezawa Y et al (2014) Proliferation and survival signaling from both Jak2-V617F and Lyn involving GSK3 and mTOR/p70S6K/4EBP1 in PVTL-1 cell line newly established from acute myeloid leukemia transformed from polycythemia vera. PLoS One 9, e84746

10. Kurosu $T$, Nagao $T$, Wu N, Oshikawa $G$ and Miura $O$ (2013) Inhibition of the PI3K/Akt/GSK3 pathway downstream of BCR/ABL, Jak2-V617F, or FLT3-ITD downregulates DNA damage-induced Chk1 activation as well as G2/M arrest and prominently enhances induction of apoptosis. PLoS One 8, e79478

11. Jain AK and Jaiswal AK (2007) GSK-3beta acts upstream of Fyn kinase in regulation of nuclear export and degradation of NF-E2 related factor 2. J Biol Chem 282, 16502-16510

12. Thomas SM and Brugge JS (1997) Cellular functions regulated by Src family kinases. Annu Rev Cell Dev Biol 13, 513-609

13. Hernandez P, Lee G, Sjoberg M and Maccioni RB (2009) Tau phosphorylation by cdk5 and Fyn in response to amyloid peptide Abeta (25-35): involvement of lipid rafts. J Alzheimers Dis 16, 149-156

14. Miyamoto Y, Yamauchi J and Tanoue A (2008) Cdk5 phosphorylation of WAVE2 regulates oligodendrocyte precursor cell migration through nonreceptor tyrosine kinase Fyn. J Neurosci 28, 8326-8337

15. Chen MC, Lin $\mathrm{H}$, Hsu FN, Huang PH, Lee GS and Wang PS (2010) Involvement of cAMP in nerve growth factortriggered p35/Cdk5 activation and differentiation in PC12 cells. Am J Physiol Cell Physiol 299, C516-C527

16. Nikolic M, Dudek H, Kwon YT, Ramos YF and Tsai LH (1996) The cdk5/p35 kinase is essential for neurite outgrowth during neuronal differentiation. Genes Dev 10, 816-825

17. Aspenstrom $P$ (1999) The Rho GTPases have multiple effects on the actin cytoskeleton. Exp Cell Res 246, 20-25

18. Ridley AJ (2006) Rho GTPases and actin dynamics in membrane protrusions and vesicle trafficking. Trends Cell Biol 16, 522-529

19. Raftopoulou M and Hall A (2004) Cell migration: Rho GTPases lead the way. Dev Biol 265, 23-32
20. Nikolic M, Chou MM, Lu W, Mayer BJ and Tsai LH (1998) The p35/Cdk5 kinase is a neuron-specific Rac effector that inhibits Pak1 activity. Nature 395, 194-198

21. Sandberg EM, Wallace TA, Godeny MD, VonDerLinden D and Sayeski PP (2004) Jak2 tyrosine kinase: a true jak of all trades? Cell Biochem Biophys 41, 207-232

22. De-Fraja C, Conti L, Magrassi L, Govoni S and Cattaneo E (1998) Members of the JAK/STAT proteins are expressed and regulated during development in the mammalian forebrain. J Neurosci Res 54, 320-330

23. Ben Haim L, Ceyzeriat K, Carrillo-de Sauvage MA et al (2015) The JAK/STAT3 pathway is a common inducer of astrocyte reactivity in Alzheimer's and Huntington's diseases. J Neurosci 35, 2817-2829

24. Kong X, Gong Z, Zhang L et al (2019) JAK2/STAT3 signaling mediates IL-6-inhibited neurogenesis of neural stem cells through DNA demethylation/methylation. Brain Behav Immun 79, 159-173

25. Spittaels K, Van den Haute C, Van Dorpe J et al (2000) Glycogen synthase kinase-3beta phosphorylates protein tau and rescues the axonopathy in the central nervous system of human four-repeat tau transgenic mice. J Biol Chem 275, 41340-41349

26. Spittaels K, Van den Haute C, Van Dorpe J et al (2002) Neonatal neuronal overexpression of glycogen synthase kinase-3 beta reduces brain size in transgenic mice. Neuroscience $113,797-808$

27. Owen R and Gordon-Weeks PR (2003) Inhibition of glycogen synthase kinase 3beta in sensory neurons in culture alters filopodia dynamics and microtubule distribution in growth cones. Mol Cell Neurosci 23, 626-637

28. Doble BW, Patel S, Wood GA, Kockeritz LK and Woodgett JR (2007) Functional redundancy of GSK-3alpha and GSK3beta in Wnt/beta-catenin signaling shown by using an allelic series of embryonic stem cell lines. Dev Cell 12, 957-971

29. Yoshimura T, Kawano Y, Arimura N, Kawabata S, Kikuchi A and Kaibuchi K (2005) GSK-3beta regulates phosphorylation of CRMP-2 and neuronal polarity. Cell 120, 137-149

30. Eom TY and Jope RS (2009) Blocked inhibitory serinephosphorylation of glycogen synthase kinase-3alpha/beta impairs in vivo neural precursor cell proliferation. Biol Psychiatry 66, 494-502

31. Seng $S, A v r a h a m ~ H K$, Jiang $S$, Venkatesh $S$ and Avraham $S$ (2006) KLHL1/MRP2 mediates neurite outgrowth in a glycogen synthase kinase 3beta-dependent manner. Mol Cell Biol 26, 8371-8384

32. Cole AR (2012) GSK3 as a sensor determining cell fate in the brain. Front Mol Neurosci 5, 4

33. Lee JH and Kim KT (2004) Induction of cyclin-dependent kinase 5 and its activator p35 through the extracellularsignal-regulated kinase and protein kinase A pathways during retinoic-acid mediated neuronal differentiation in human neuroblastoma SK-N-BE(2)C cells. J Neurochem 91, 634-647

34. Bibb JA, Chen J, Taylor JR et al (2001) Effects of chronic exposure to cocaine are regulated by the neuronal protein Cdk5. Nature 410, 376-380

35. Kim SY, Han YM, Oh M et al (2015) DUSP4 regulates neuronal differentiation and calcium homeostasis by modulating ERK1/2 phosphorylation. Stem Cells Dev 24, 686-700 